\title{
Fatores que influenciam a não utilização das Academias ao Ar Livre na cidade de Apucarana-PR
}

\section{Factors that influence the non-use of Open Fitness Zones in the city of Apucarana-PR}

\author{
Josimar Florêncio de Morais ${ }^{1}$ \\ Orcid: https://orcid.org/0000-0001-9194-7379 \\ Jymmys Lopes Dos Santos 3 \\ Orcid: https://orcid.org/0000-0003-1433-2041 \\ Frederico Barros Costa ${ }^{5}$ \\ Orcid: https://orcid.org/0000-0002-7117-4185
}

\author{
Lucio Marques Vieira-Souza ${ }^{2}$ \\ Orcid: https://orcid.org/0000-0002-5721-0725
}

Cássio Murilo Almeida Lima Júnior ${ }^{4}$
Orcid: https://orcid.org/0000-0002-1603-7225

Fábio José Antônio Da Silva ${ }^{6}$

Orcid: https://orcid.org/0000-0002-5881-6438

\begin{abstract}
Resumo
Introdução: As Academias ao Ar Livre são espaços públicos que objetivam promover atividade física de forma gratuita à população, tendo sido instaladas no Brasil desde o ano de 2005, baseado no modelo chinês. Já na cidade de Apucarana, seu início é datado de 2006, havendo, atualmente, 42 delas. Objetivo: O presente trabalho objetiva conhecer os fatores pelos quais a população de Apucarana utiliza as Academias ao Ar Livre. Materiais e Métodos: Foi utilizada uma abordagem do tipo quantitativa, cuja coleta dos dados foi realizada através da utilização de questionário semiestruturado contendo perguntas fechadas aplicado a 384 pessoas da localidade onde as Academias ao Ar Livre estão instaladas. A análise e os gráficos foram elaborados através do Software Epi Info ${ }^{\mathrm{TM}}$ 7.2. Resultados: Os motivos que levaram a população a utilizar a Academias ao Ar Livre foram: Promoção à saúde $(38,31 \%)$, Lazer (25,87\%), Qualidade de vida (20,40\%), Estética (7,46\%), Prazer (4,48\%), Outros (2,49\%), Interação Social (1\%). Conclusão: É necessário o poder público manter as Academias ao Ar Livre em funcionamento, pois este tipo de política se estabelece em longo prazo, haja vista um número significativo de usuários, assim como aqueles que não usam, mas tem intenção de utilizar futuramente.

Palavras-chave: espaço público; população; atividade física.
\end{abstract}

\section{Abstract}

Introduction: Open Fitness Zones are public spaces that aim to promote free physical activity to the population, which have been implemented in Brazil since 2005, based on the Chinese model. In the city of Apucarana, Open Fitness Zones began to be implemented in 2006, and there are currently 42 of them. Objective: The present work aims to understand the factors by which the population of Apucarana uses Open Fitness Zones. Materials and Methods: A quantitative approach was used, whose data collection was carried out through the use of a semi-structured questionnaire containing closed

\footnotetext{
${ }^{1}$ Departamento de Ensino e Pesquisa, Autarquia Municipal de Saúde, Apucarana-PR, Brasil. E-mail: maktubsalaam@hotmail.com

${ }^{2}$ Faculdade de Educação Física, Universidade do Estado de Minas Gerais-UEMG, Campus Passos - MG, Brasil. / Programa de Pós-Graduação em Educação Física (PPGEF), Universidade Federal de Sergipe-UFS, São Cristóvão-SE, Brasil. E-mail: profedf.luciomarkes@gmail.com

${ }^{3}$ Programa de Pós-Graduação em Educação Física (PPGEF), Universidade Federal de Sergipe-UFS, São Cristóvão-SE, Brasil. E-mail: jymmyslopes@yahoo.com.br

${ }^{4}$ Programa de Pós-Graduação em Saúde e Ambiente, Universidade Tiradentes-UNIT, Aracaju-SE, Brasil. / Laboratório de Biociências da Motricidade Humana (LABIMH), Universidade Tiradentes-UNIT, Aracaju-SE, Brasil. E-mail: murilo.cassio@hotmail.com

${ }^{5}$ Laboratório de Biociências da Motricidade Humana (LABIMH), Universidade Tiradentes-UNIT, Aracaju-SE, Brasil. Email: costafredericob@gmail.com

${ }^{6}$ Programa de Residência em Atenção Básica/Saúde da Família da Autarquia Municipal de Saúde de Apucarana-PR, Brasil. E-mail: fjas81@hotmail.com
} 
questions applied to 384 people from neighborhoods where Open Fitness Zones are installed. Analyses and graphs were performed using the Epi Info тм 7.2 software. Results: The reasons that led the population to use OFZ were: Health promotion (38.31\%), Leisure (25.87\%), Quality of life (20.40\%), Aesthetics (7.46 \%), Pleasure (4.48\%), Others (2.49\%), Social Interaction (1\%). Conclusion: Public authorities should keep Open Fitness Zones in operation, as this type of policy is established in the long term, considering the significant number of users, as well as those who do not use them, but intend to use them in the future.

Keywords: public space; population; physical activity.

\section{Introdução}

Todo movimento corporal produzido que resulte em gasto energético realizado pela musculatura esquelética, sejam lutas, jogos ou danças, esportes, atividades de lazer ou trajetos, que tenham aspectos biopsicossociais relacionados ao comportamento ou cultural, pode ser compreendido como atividade física ${ }^{1}$.

No momento atual em que vivemos, comprova-se que pessoas mais ativas tem menos limitações físicas, e que a relação entre uma vida ativa tem sido associada a vários estudos mostrando que há um aumento na longevidade ${ }^{2-3}$. Observou-se que manter um estilo de vida ativo favorece $o$ processo de envelhecimento de uma forma positiva, bem como é possível entender que a prática regular de atividade física desenvolve várias capacidades físicas ${ }^{4}$.

Em virtude disso, compreende-se que a Academia ao Ar Livre (AAL), como Política Pública de Esporte e Lazer, destinada a incentivar à prática de exercícios físicos pode ser vista como uma ferramenta que visa tratar da saúde da população de uma forma integral, já que o exercício tem efeitos fisiológicos que potencializam a melhora da saúde do indivíduo e minimizam efeitos deletérios, sendo também um espaço de interação social $^{2,3,5}$.

Estes locais oferecem um baixo índice de lesões para seus praticantes ${ }^{6}$. Em um trabalho realizado com o intuito de observar parâmetros morfofuncionais constatou-se que não houve melhorias com a prática de atividade física na $\mathrm{AAL}$, porém alterações cardiovasculares foram apontadas como fator de contribuição para diminuição da pressão $\operatorname{arterial}^{7}$.

Compreendem-se as AAL como espaços públicos, pois são instaladas em parques, praças ou complexos esportivos ${ }^{8}$. Neste sentido o uso destes espaços tem sido uma ferramenta muito utilizada para atividades esportivas ou simples momento de relaxamento 9 . Pensando nisso, há a necessidade de reorganizar e desfragmentar estes espaços, pois são vistos como promotores de saúde e que potencializam a qualidade de vida, sendo locais de coletividade e, possivelmente, relaciona-se à falta destes com a pouca prática de atividade física pelas pessoas no lazer, os quais devem propiciar às diversas práticas corporais ${ }^{10}$. De tal forma quando pensamos em incentivar as práticas corporais devemos nos ater em formas de planejar ações que garantam a segurança e o conforto de quem pratica, ou seja, os locais devem ter condições estabelecidas de acessibilidade e convivência a fim de garantir seu usufruto. Outra estratégia que pode ser utilizada é a relação intersetorial, aproveitando estes espaços como verdadeiros lugares de convivência social, e tornando um desafio aos gestores do SUS na manutenção deles, utilizando-os para praticar exercícios ${ }^{11-12}$.

Outra condição elementar que talvez impeça a frequente utilização das AAL seria a ausência do Profissional de Educação Física, muito embora haja recomendações sobre tal necessidade, geralmente não se nota tal presença naquela academia $^{13}$. A maioria das entidades públicas preferem instalar estes equipamentos próximos as Unidades Básicas de Saúde onde há um grande número de pessoas portadoras de Doenças 
Crônicas Não Transmissíveis (DCNTs) como hipertensão e diabetes, pois muitos profissionais afirmam que, havendo um espaço que favoreça uma terapia não medicamentosa para estas ou outras doenças, favorecerá sua recuperação bem como promoção da saúde, sem grandes investimentos por parte dos gestores ${ }^{14}$. Pessoas que não têm o hábito de praticar atividade física quando recebem orientação tendem a melhorar a sua regularidade, e aquelas que já são regulares, podem aumentar esta prática ainda mais ${ }^{14}$. Devese ressaltar sobre a importância da contratação de profissionais especializados a fim de maximizar o uso do equipamento público e potencializar os resultados adquiridos pela população usuária ${ }^{15}$.

A AAL teve início no Brasil, como projeto piloto, dentro do Programa Maringá Saudável, no ano de 2005, tendo então sua primeira academia instalada em 2006. Estes equipamentos foram adaptados por um engenheiro civil com formação em Educação Física, inspiradas no modelo Chinês, que visava fomentar a prática de atividade física na população daquele país, principalmente devido à vinda dos jogos olímpicos ${ }^{16}$. Atualmente na cidade de Apucarana existem aproximadamente 42 (quarenta e duas) AAL espalhadas por vários bairros, de acordo com o IDEPPLAN (Instituto de Desenvolvimento, Pesquisa e Planejamento de Apucarana). Não foram encontrados registros oficiais de quando se deu a primeira instalação destas academias em Apucarana. Portanto, o presente trabalho objetiva conhecer os fatores pelos quais a população de Apucarana utiliza as Academias ao Ar Livre.

\section{Materiais e Métodos}

\section{Amostra e tipo de estudo}

A metodologia utilizada foi uma abordagem quantitativa descritiva exploratória através da aplicação de questionário semiestruturado contendo perguntas fechadas, numa amostra obtida através de cálculo amostral baseado numa população de 131.571 mil habitantes ${ }^{17}$. Obteve-se 384 amostras (Fluxograma). Antes da entrevista foi entregue e lido o Termo de Consentimento Livre Esclarecido (TCLE) aos participantes da pesquisa, no qual apresenta explicações quanto ao procedimento, finalidade do estudo e seus possíveis riscos e benefícios. Este estudo está em conformidade com a Resolução $n^{\circ} 466$, de 12 de dezembro de 2012 do Conselho Nacional de Saúde, a qual estabelece as diretrizes e normas regulamentadoras de pesquisas envolvendo seres humanos, e passou por avaliação no Comitê de Ética em Pesquisa com Seres Humanos (CETi) da Faculdade de Apucarana sob o parecer número: 1957080. 
Morais FM, Lucio Marques Vieira-Souza LMV, Santos JL, Lima Júnior CMA, Costa FB, Silva FJA

Fluxograma 1. Amostra de entrevistados

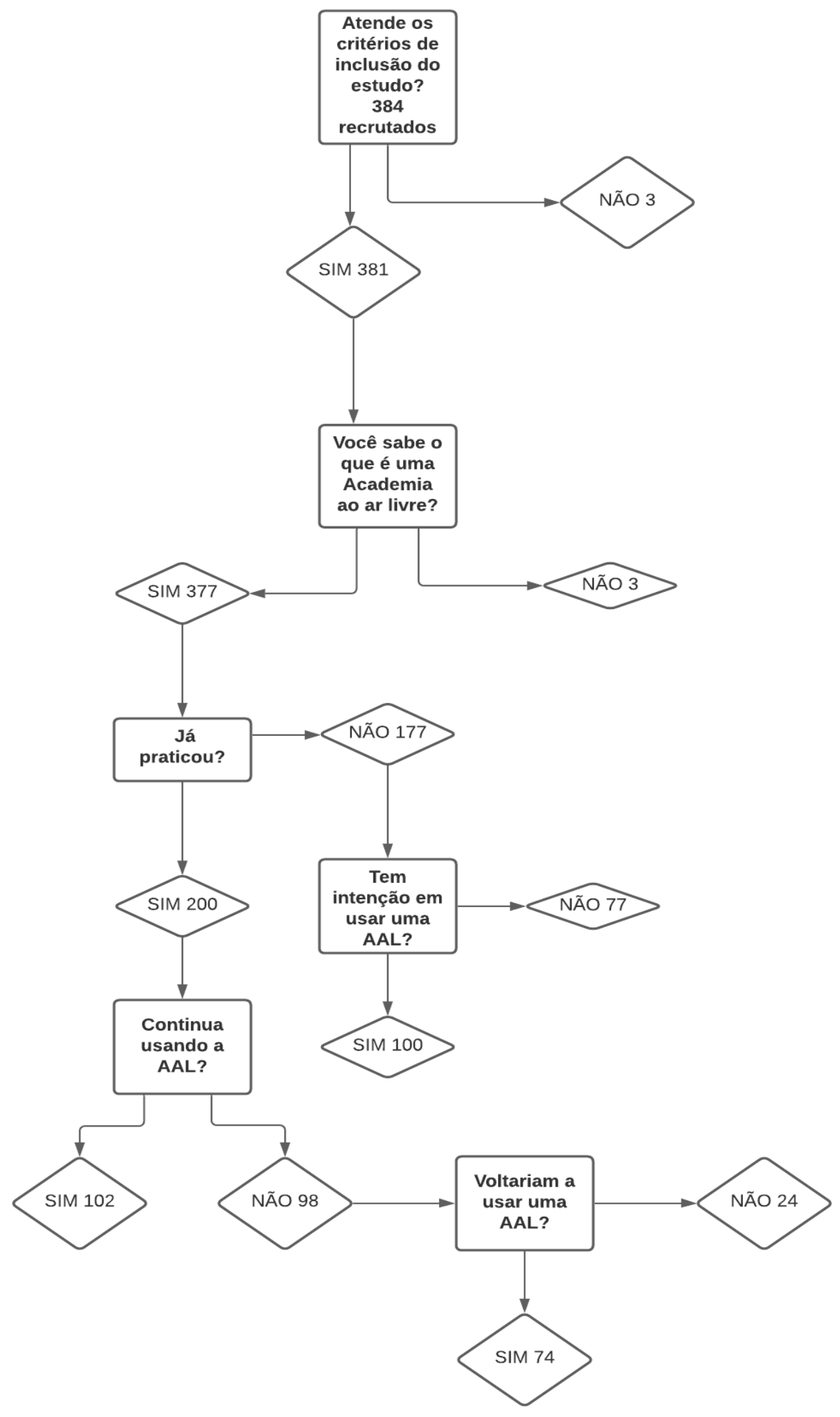

\section{Delineamento da pesquisa}


Os entrevistados foram escolhidos de forma aleatória em diferentes locais como pontos de ônibus, estabelecimentos comerciais, na rua e em praças e o questionário aplicado em horário comercial (08h às $18 \mathrm{~h})$.

\section{Critérios de Inclusão e Exclusão}

Os entrevistados foram escolhidos de forma aleatória, em horário comercial, entrevistados em pontos de ônibus, estabelecimento comerciais, na rua, em praças.

\section{Procedimentos}

Os questionários foram aplicados com moradores da área de abrangência das Academias ao Ar Livre da cidade de Apucarana/PR.

Como critérios de inclusão e exclusão:

\section{Resultados}

A amostra foi composta por 384 indivíduos dos quais três foram excluídos pelo critério de exclusão, sendo a primeira pergunta da pesquisa referente ao conhecimento que o indivíduo tinha sobre o que é uma Academia ao Ar Livre (AAL). Se o participante respondesse que não sabia o que era este equipamento, a pesquisa com este participante terminava a partir deste ponto, sendo coletado apenas este dado. Sendo assim dos 381, apenas $0,79 \%$ responderam que não sabiam, restando 99,21\% afirmando que conheciam, com os quais foi dada a sequência nas outras variáveis da pesquisa. Da amostra total $61,94 \%$ eram mulheres e $38,06 \%$ homens.

A média de idade dos participantes foi de 42 anos (desvio-padrão - DP = 17,31). Relacionado à escolaridade, $9,19 \%$ tinham o Ensino Fundamental completo, $31,23 \%$ o Ensino Fundamental incompleto, 29,13\% Ensino Médio Completo, 14,44\% Ensino Médio Incompleto, 6,56\% Superior Completo, 7,35\% Superior Incompleto e $2,10 \%$ sem escolaridade ou analfabeto.

Tabela 1: Características sociodemográficas da amostra do estudo ( $\mathrm{n}=384)$.

\begin{tabular}{lcc}
\hline Sexo & Variáveis & Frequência simples \\
& Homens & $38 \%$ \\
& Mulheres & $62 \%$
\end{tabular}

Idade
Anos
$42 \pm 17,31$

\section{Escolaridade}

$\begin{array}{cc}\text { Ensino Fundamental Completo } & 9,19 \% \\ \text { Ensino Fundamental Incompleto } & 31,23 \% \\ \text { Ensino Médio Completo } & 23,13 \% \\ \text { Ensino Médio Incompleto } & 14,44 \% \\ \text { Ensino Superior Completo } & 6,56 \% \\ \text { Ensino Superior Incompleto } & 7,35 \% \\ \text { Sem escolaridade ou analfabeto } & 2,10 \%\end{array}$

Fonte: Autoria própria.

No que concerne à ocupação, $10,76 \%$ responderam que eram aposentados, $3,67 \%$ desempregados, $13,65 \%$ do lar (relatado por mulheres), $65,35 \%$ empregados e $6,56 \%$ estudantes. Em relação à renda: $37,53 \%$ responderam ter uma renda individual de até um saláriomínimo; $51,18 \%$ entre 1 a $3 ; 4,46 \%$ entre 3 a 6; e 6,82\% dos participantes declararam não possuir renda. Quando cruzados os dados sobre renda e uso da AAL os seguintes resultados foram obtidos em relação àqueles que mais fazem uso de acordo com a renda individual, como pode ser visto na figura abaixo: 
Figura 1. Uso da renda ALL/Renda.

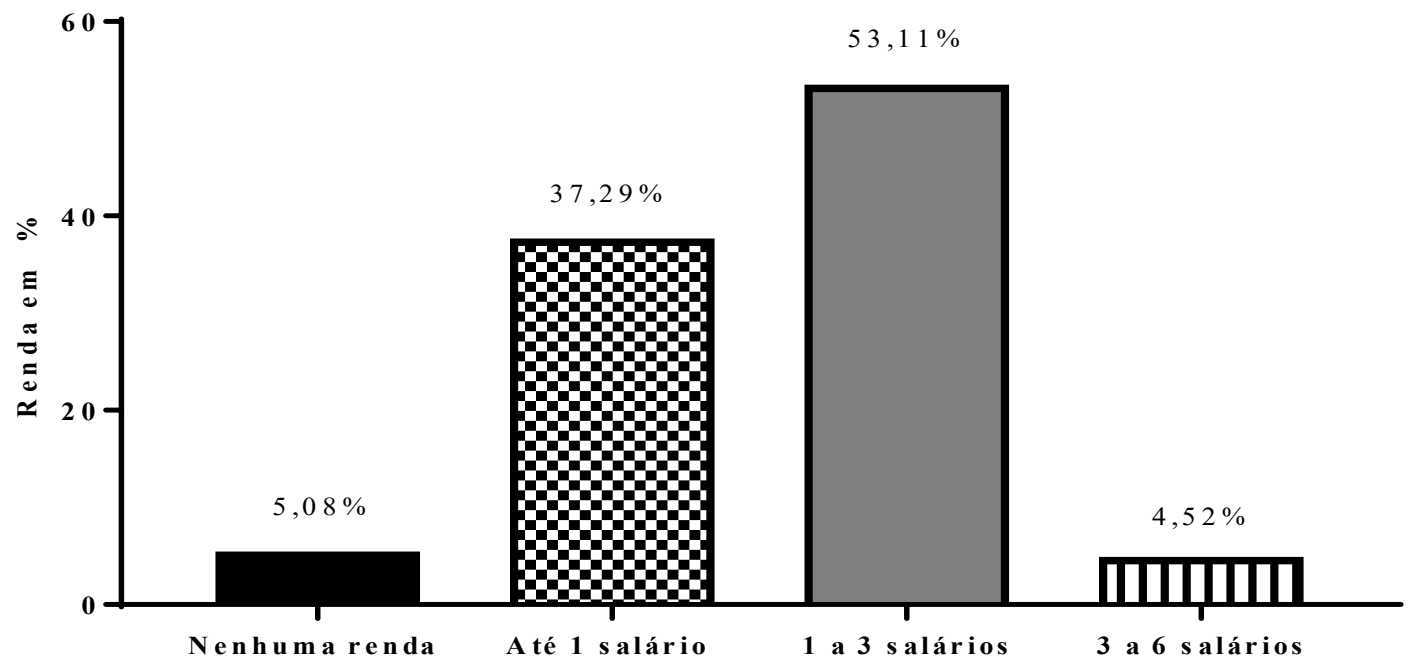

Quanto à utilização da AAL, $46,83 \%$ responderam que nunca utilizaram e $53,17 \%$, já utilizaram. Entre aqueles que não utilizaram as principais razões estão na figura abaixo:

Figura 2. Por que nunca utilizou?

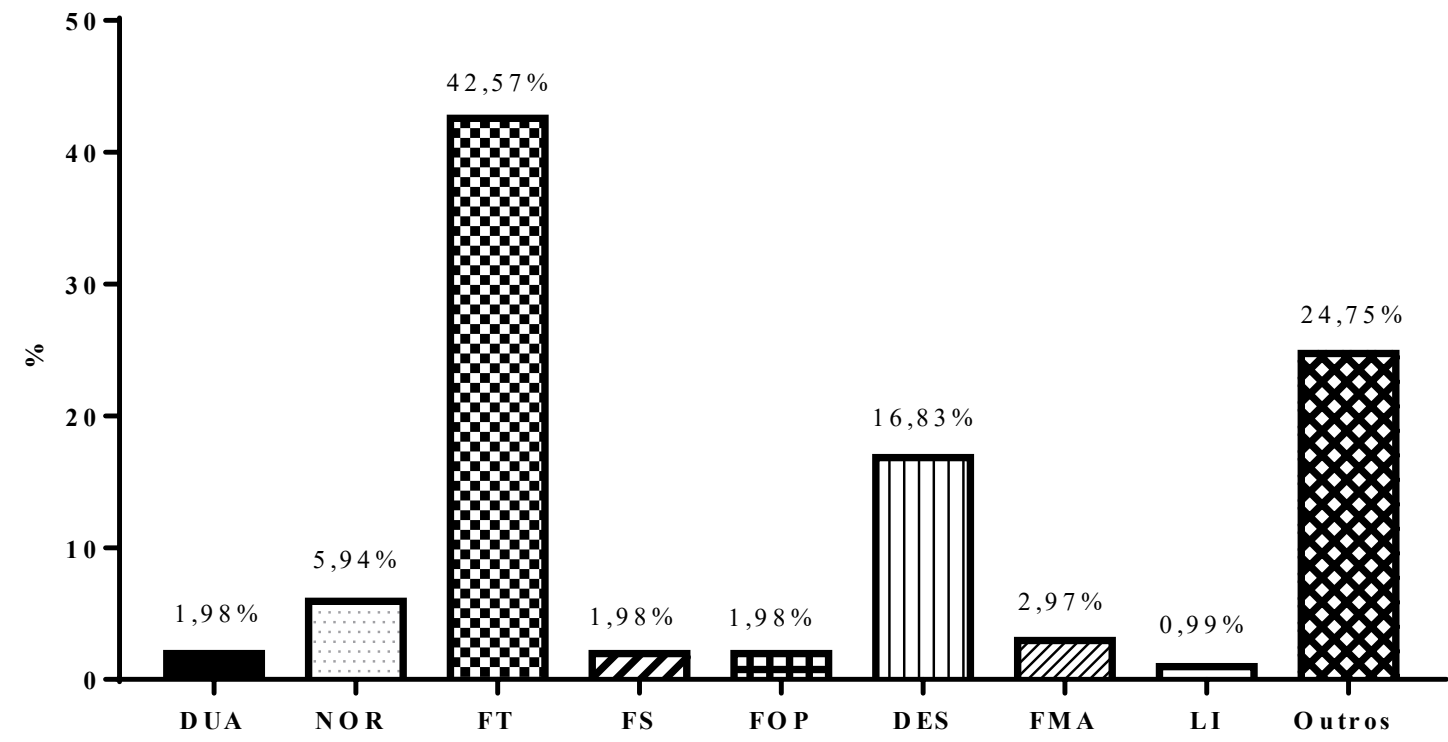

Legenda: VEP: vergonha de exercitar-se em público; LI: local para idosos; FT: falta de tempo; FS: falta de segurança; FOP: falta de orientação profissional; DES: desmotivação; FMA: falta de manutenção dos aparelhos; FL: falta de limpeza e Outros. 
Quanto àqueles que nunca utilizaram, quando perguntados se tinham intenção de utilizar: $54,8 \%$ responderam Sim; 38,42\% responderam Não; e 6,78\% responderam talvez. Para os entrevistados que responderam que utilizaram ao menos uma vez, os objetivos estão na figura seguinte:

Figura 3. Qual o objetivo?

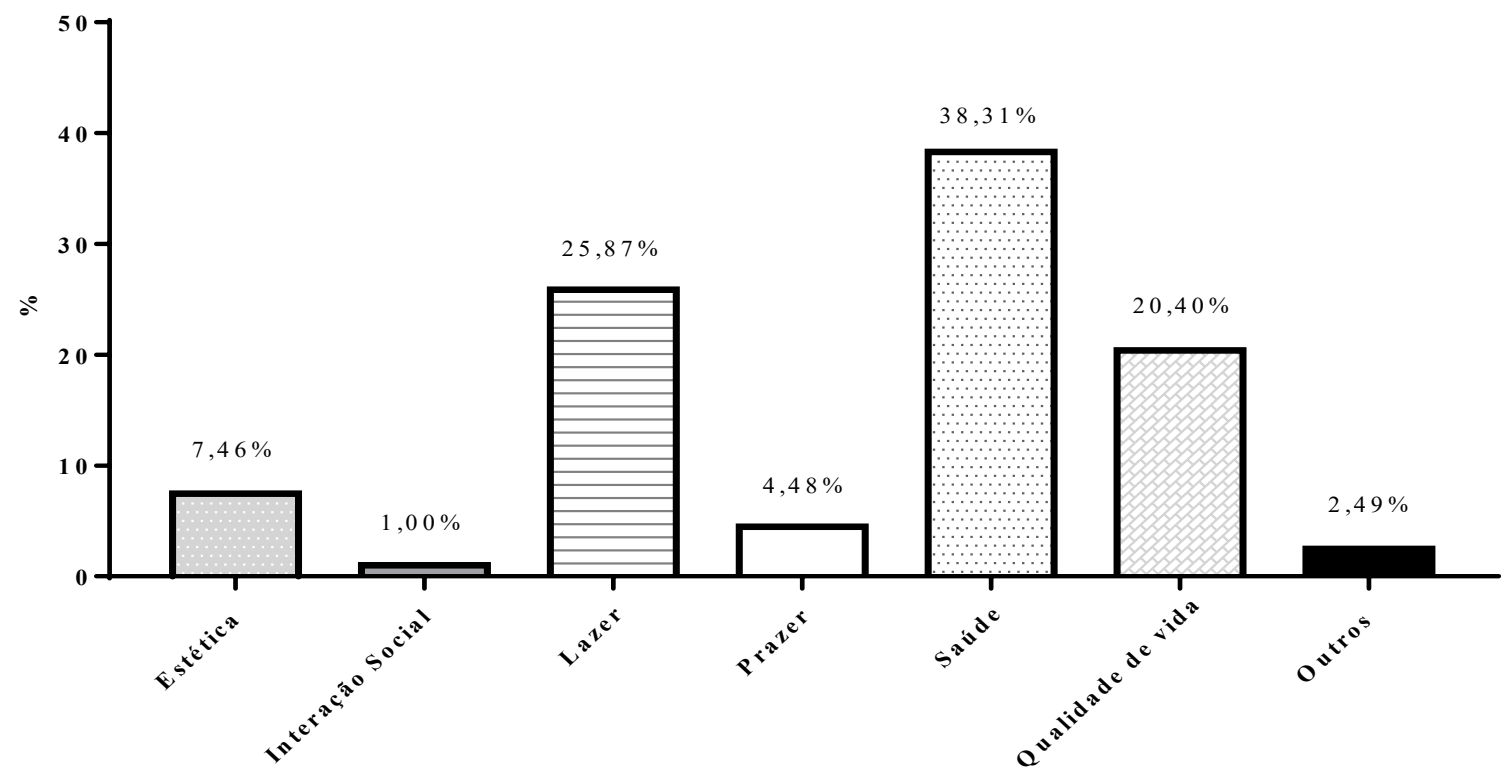

Destes que disseram que utilizaram, $49,75 \%$ continuam utilizando e $50,25 \%$, deixaram de usar. Os principais motivos para o desuso estão relacionados na figura abaixo:

Figura 4. Por que usou e deixou de usar?

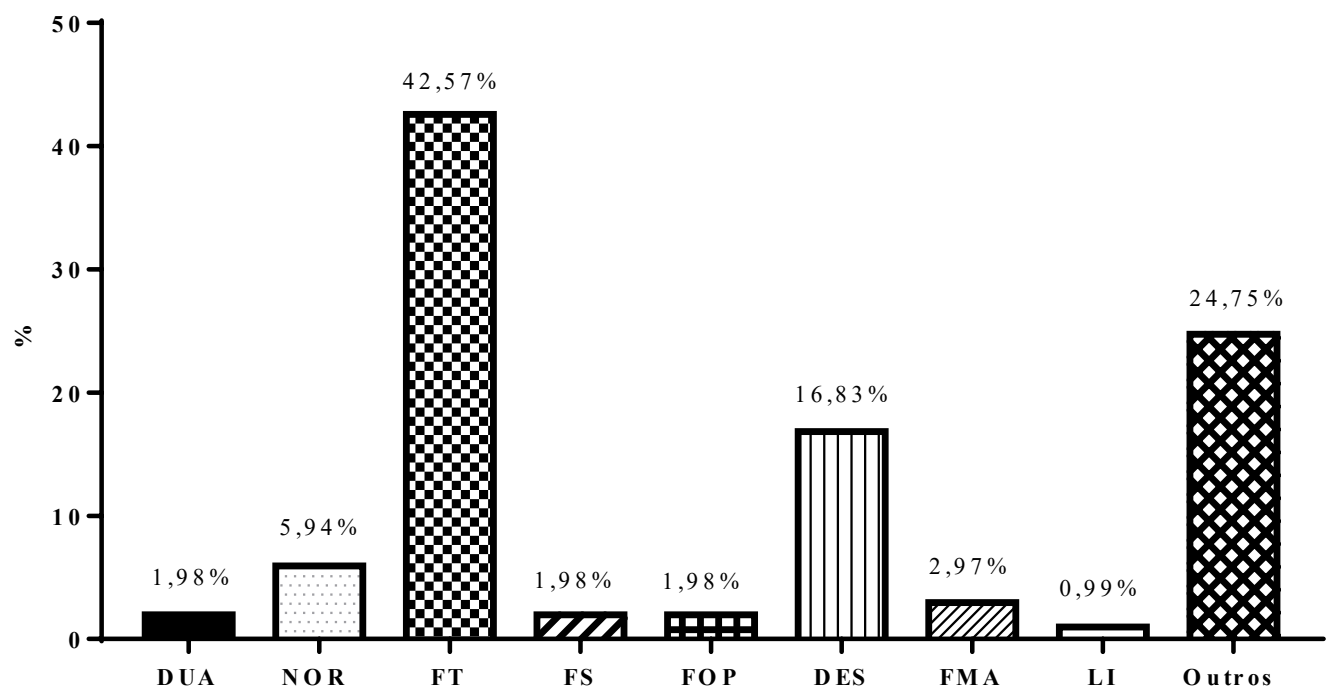

Legenda: DUA: dificuldade de utilizar os aparelhos; NOR: não obteve resultados; FT: falta de tempo; FS: falta de segurança; FOP: falta de orientação profissional; DES: desmotivação; FMA: falta de manutenção dos aparelhos; LI: local para idosos; e outros. 
Ainda para estes indivíduos que já usaram a AAL e deixaram de usar, foi perguntado se voltariam a usar. As respostas foram as seguintes: $\operatorname{Sim} 75,25 \%$, Não $14,85 \%$ e talvez $9,90 \%$.

No tocante à frequência semanal os resultados mostraram que: $28,36 \%$ utilizam duas vezes por semana; $27,36 \%$ uma vez; $24,88 \%$ três vezes; $14,93 \%$ quatro vezes; $3,98 \%$ cinco vezes; e $0,50 \%$ utilizam sete vezes. Referente ao tempo de uso da AAL, podemos apontar os seguintes resultados: $38,21 \%$ utilizam por 30 minutos; $20,40 \%$ por 60 minutos; $18,91 \%$ por 40 minutos; $17,91 \%$ por 15 minutos; $3,98 \%$ por 50 minutos; e $0,50 \%$ por 20 minutos. Com relação ao tempo semanal a figura abaixo mostra que $77,11 \%$ usaram menos do que 150 minutos semanais e apenas $22,89 \%$ atingiram este tempo.

Figura 5. Tempo semanal acumulado.

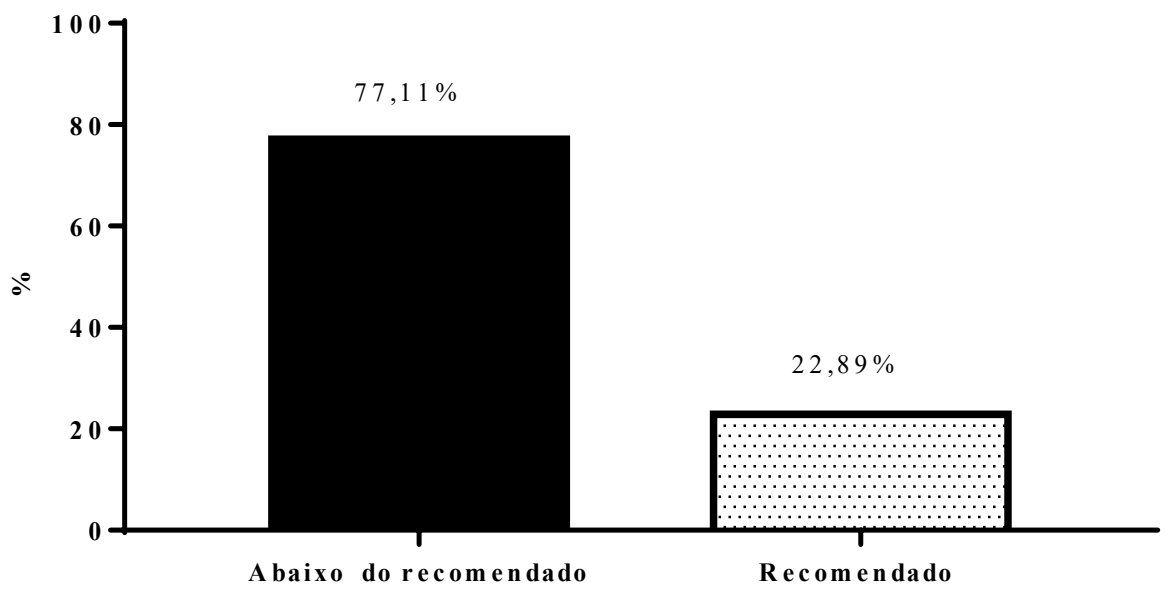

\section{Discussão}

O presente estudo questionou uma amostra da população da cidade de Apucarana/PR sobre os principais fatores que os influenciam a usarem as Academias ao Ar Livre (AAL). Os principais achados foram três fatores que estão intimamente relacionados e que predominaram na pesquisa, sendo estes os que mais influenciaram as pessoas a usar a AAL: Promoção da Saúde, Qualidade de Vida e Lazer. Sendo assim, é importante ressaltar que a Promoção da Saúde, explicita na Carta de Ottawa de 1986, é um processo para tornar capaz a população a atuar na melhoria de sua qualidade de vida, e sobretudo o lazer deveria ser uma fonte de saúde para as pessoas. $\mathrm{O}$ documento enfatiza que a promoção da saúde não é responsabilidade apenas do poder público $^{18}$. Logo a percepção das pessoas em relação à utilização da AAL buscando os fatores supracitados demonstra uma visão ampliada e importante na melhoraria da sua saúde. Apesar das pessoas terem esta percepção, os níveis de inatividade continuam elevados, sendo necessárias ações de vários setores da sociedade para melhorar este quadro ${ }^{19}$. Também é importante lembrar que o lazer está presente na constituição de 1988 em dois momentos, no artigo sexto como um direito social e no artigo 217 parágrafo terceiro deixando claro que o poder público incentivará o lazer como promoção social $^{20}$.

Neste sentido, a criação e manutenção de espaços públicos que 
promovam o lazer, contribuirão para a promoção da saúde, que está diretamente ligada a qualidade de vida, assim, foi criada em 2006 a PNPS (Política Nacional de Promoção da Saúde) a qual tem em um dos seus objetivos promover a melhoria da qualidade de vida ${ }^{19}$. A qualidade de vida pode ser definida em termos de distância entre as expectativas individuais e a realidade ${ }^{21}$.

Desta forma quando há espaços que promovem a prática de atividade física para a população, esta distância pode ser diminuída e a realidade alcançada. Neste sentido alguns fatores que influenciaram o uso da AAL como: estética, prazer e interação social, que aparecem em menor porcentagem, mas que foram elencados pela população também podem se tornar realidade. No entanto para que alguns dos objetivos supracitados sejam alcançados há um fator de extrema importância ao qual precisamos nos ater, o tempo de uso semanal. Quando observamos esta variável vemos que apenas $22,89 \%$ utilizaram por 150 minutos, enquanto $77,11 \%$ não atingiu este patamar. Recomenda-se que adultos para terem benefícios à saúde façam $\underline{300}$ minutos de atividade física aeróbia durante a semana com intensidade moderada a intensa ou 75 minutos de atividade física aeróbia vigorosa ou combinação de ambas, mantê-las pode retardar declínios funcionais ${ }^{22}$.

Quanto à participação em programas de atividade física, um estudo que avaliou um programa de atividade física na cidade do Recife/PE, encontrou que $65,7 \%$ dos usuários responderam participar por causa da saúde, enquanto apenas $0,7 \%$ participam por lazer, talvez este último seja pelo fato de haver outros espaços que possam ser utilizados para tal atividade naquela cidade $^{15}$. Durante $o$ contato com os participantes da pesquisa na cidade de Apucarana houve muitos relatos sobre a falta de espaços que incentivam esta prática. Apesar das duas populações terem uma percepção parecida sobre a atividade física como algo benéfico para promover a saúde, há uma grande diferença em relação à utilização de espaços deste tipo como prática de lazer.

Em outro estudo realizado na cidade de Recife que buscou identificar a procura por programas de promoção da saúde por idosos, encontrou-se que $84,2 \%$ procuram estes programas para melhorar a saúde, $47,5 \%$ disseram que participa para melhorar a autoestima e relaxar. Se considerarmos que o lazer é usado como forma de relaxamento, é possível associar que esta população indiretamente busca estes espaços para tal finalidade ${ }^{23-24}$.

Em estudo sobre a adesão a prática de atividade física tiveram resultados que indicam: a ordem médica, o lazer, a qualidade de vida, estética e condicionamento físico como os principais motivos para esta prática ${ }^{25}$. A busca por estes espaços também pode ser entendida de acordo com aspectos socioeconômicos, sendo que o presente estudo encontrou resultados semelhantes aos do estudo realizado por outro autor ${ }^{19}$.

O principal motivo alegado pelos participantes para não usar a $\mathrm{AAL}$ foi à falta de tempo, tanto para aqueles que nunca usaram como para quem usou e deixou de usar, seguido por outros motivos (doença, afastamento por recomendação médica, gestação, preguiça, falta de orientação profissional e desmotivação). Como a maior parte da amostra são de pessoas empregadas, quando foram relacionados à ocupação com a falta de tempo respectivamente, $81,33 \%$ dos que responderam não usar por falta de tempo estão nesta condição. Entre os que usaram e deixaram de usar, 72,09\% também estão empregadas. Apesar da maior parte da amostra ser de empregados, é preciso discorrer sobre a relação do tempo e do modo de viver no nosso dia a dia, ou seja, até que ponto é a falta de tempo ou a falta de organização para atitudes saudáveis? $\mathrm{O}$ tempo está relacionado aos dias, horas, minutos e com o passado, presente e futuro, mas também com o momento ou ocasião apropriada para que algo se 
realize $^{15}$. O modelo de organização Taylorista ainda muito presente na sociedade, destrói a individualidade humana, assim os indivíduos utilizam o tempo especializado no trabalho organizando-se demasiadamente para $\mathrm{o}$ mesmo e deixa de lado outras atividades ${ }^{26}$. A falta de tempo é uma questão de prioridade, deste modo um programa de atividade física deve ser atraente suficiente para derrubar este fator; já a falta de energia parece estar mais relacionada a uma questão mental do que física, sendo um motivo a mais para buscar praticar atividade física a partir do conhecimento dos benefícios; quanto à desmotivação esta é soma de dois fatores, ou seja, o cansaço com a dedicação do tempo em outras atividades $^{24}$. Sendo assim a orientação profissional foi reportada como um fator importante para adesão, onde os usuários disseram $(57,5 \%)$ que gostariam de receber atenção do professor e $(62,5 \%)$ receber incentivos $^{23}$. Logo a presença de um profissional para explicar os benefícios e prescrever o exercício de forma que o usuário aproveite este benefício é de extrema importância. A fragmentação e a individualização do trabalho levam há novos tempos laborais, assim o indivíduo tem menos tempo social, ou seja, deixa de lado a família, o lazer, a educação e até mesmo o descanso. Existe uma ampliação velada do tempo de trabalho, sendo assim, cada vez mais o trabalhador estaria a serviço da empresa, levando tarefas para casa, diminuindo o seu tempo social ${ }^{26}$.

Como possível limitação do estudo fica a possibilidade de pesquisas futuras em caráter longitudinal, e avaliando a importância ou não da presença do Profissional de Educação Física nesses espações.

\section{Conclusão}

Conforme os dados apresentados, boa parte da população estudada ainda não faz uso devido das AAL, portanto sugerese que existe a necessidade do poder público manter as AAL em funcionamento propiciando espaços públicos que ofertem a prática de hábitos saudáveis. Este tipo de política se estabelece em longo prazo, haja vista um número significativo de usuários destas AAL, assim como aqueles que reportaram não usar, mas que têm intenção de utilizar futuramente, sobretudo pelo fato de que os motivos alegados para praticar atividade física pela população vão de encontro a agenda de órgãos internacionais os quais incentivam um comportamento ativo como meio de promover a saúde e diminuir gastos públicos.

\section{Referências}

1. Silva CL, Souza MF, Filho SR, Silva, LF, Rigoni ACC. Atividade física de lazer e saúde: uma revisão sistemática. Mudanças - Psicologia da Saúde. 2017; 25(1): 54-67.

2. Carvalho RBC, Madruga VA. Envelhecimento e prática de atividade física: a influência do gênero. Motriz, Rio Claro. 2011; 17(2): 328-337.

3. Silva LS, Santos Ribeiro, DSS. Treinamento funcional: contribuições para a qualidade de vida de moradores da área rural de Paripiranga/BA. Ciência em Movimento Reabilitação e Saúde. 2020; 43(22): 123-137.

4. Mari FR, Alves GG, Aerts DRG, Camara S. O processo de envelhecimento e a saúde: o que pensam as pessoas de meia-idade sobre o tema. Rev bras geriatr gerontol. 2016;19(1): 35-44. 
5. Camboim FEF, Nóbrega MO, Davim RMB, Camboim JCA, Nunes RMV, Oliveira SX. Benefícios da atividade física na terceira idade para a qualidade de vida. Rev enferm UFPE [online]. 2017; 11(6): 2415-22.

6. Silva AT, Firmino RC, Albérico CO, Reis RS. Fatores associados à ocorrência de lesões durante a prática de atividade física em academias ao ar livre. Revista Brasileira de Medicina do Esporte. 2016; 22(4): 267-271.

7. Esteves, JVDC, Andreato LV, Pastório JJ, Versuti JKB, Almeida HDC, de Moraes SMF. $\mathrm{O}$ uso de academias da terceira idade por idosos modifica parâmetros morfofuncionais? Acta Scientiarum. 2012; 34(1): 31-38.

8. Costa B, Freitas C, Silva K. Atividade física e uso de equipamentos entre usuários de duas Academias ao Ar Livre. Revista Brasileira de Atividade Física \& Saúde. 2016; 21(1): 29-38.

9. Sanches SM, Rubio K. Prática esportiva como ferramenta educacional: trabalhando valores e a resiliência. Educação e Pesquisa. 2011; 37(4): 825-842.

10. Iepsen A, Silva M. Perfil dos frequentadores das academias ao ar livre da cidade de Pelotas - RS. Rev Bra Atividade Física \& Saúde. 2015; 20(4): 413-424.

11. Azevedo ED, Pelicioni MCF, Westphal MF. Práticas intersetoriais nas políticas públicas de promoção de saúde. Physis: Rev de Saúde Coletiva. 2012; 22 (4): 1333-1356.

12. Becker L, Gonçalves P, Reis R. Programas de promoção da atividade física no Sistema Único de Saúde brasileiro: revisão sistemática. Rev Bras Ativ Fís Saúde. 2016; 21(2):110-122.

13. Costa B, Freitas C, Silva K. Atividade física e uso de equipamentos entre usuários de duas Academias ao Ar Livre. Rev Bra de Atividade Física e Saúde. 2016; 21(1): 29-38.

14. Malta DC, Castro AMD, Gosch CS, Cruz DKA, Bressan A, Nogueira JD, et al. Política Nacional de Promoção da Saúde e a agenda da atividade física no contexto do SUS National. Epidemiol. Serv. Saúde. 2009; 18(1): 79-86.

15. Hallal PC, Tenório MCM, Tassitano RM, Reis RS, Carvalho YM, Cruz DKA, et al. Avaliação do programa de promoção da atividade física Academia da Cidade de Recife, Pernambuco, Brasil : percepções de usuários e não usuários. Cad. Saúde Pública. 2010; 26(1): 70-78.

16. Veríssimo, LCG. O processo de construção em rede de políticas públicas na perspectiva da promoção da saúde: a experiência do município de Maringá-PR. [Dissertação de Mestrado] Campinas: Faculdade de Ciências Médicas/Departamento de Medicina Preventiva e Social - Unicamp; 2011.

17. IBGE - Instituto Brasileiro de Geografia e Estatística. Censo demográfico: Apucarana Brasil; 2017

18. WHO. Carta de Ottawa. In Ministério da Saúde/FIOCRUZ. Ponto Promoção da Saúde. Cartas de Ottawa, Adelaide, Sundsvall, Santa Fé de Bogotá. Ministério da Saúde/IEC; 1986. $11-18$ 
19. Ribeiro RM, Tribess S, Santos ASD, Pinto LLT, Ribeiro MDCL, Roza LB, et al. Barreira no engajamento de idosos em serviços públicos promoção de atividade física. Ciência \& Saúde Coletiva. 2015; 20(3): 739-749.

20. Brasil, Constituição (1988). Constituição da República Federativa do Brasil. Brasília (DF): Senado Federal; 1988.

21. Pereira EF, Teixeira CS, Santos A. Qualidade de vida: abordagens, conceitos e avaliação. Rev. bras. Educ. Fís. Esporte. 2012; 26(2): 241-50.

22. Hammami A, Harrabi B, Mohr M, Krustrup P. Physical activity and coronavirus disease 2019 (COVID-19): specific recommendations for home-based physical training. Manag Sport Leis. 2020; 20(0):1-6

23. Freitas CMSM, Santiago MDS, Viana AT, Leão, A. C., \& Freyre, C. Aspectos motivacionais que influenciam a adesão e manutenção de idosos em programas de exercícios físicos. Rev. Bras. Cineantropm Desempenho Hum. 2007; 9(1): 92-100.

24. Ribeiro JAB, Cavall AS, Cavalli MO, Pogorzelski LV, Prestes MR, Ricardo LIC. Adesão de Idosos a Programas de Atividade Física: Motivação e Significância. Rev. Bras. Ciênc. Esporte. 2012; 34(4) 969-984.

25. Zamai CA, Bavoso D, Rodrigues AA, Barbosa JAS. Motivos de adesão, manutenção e resultados alcançados através da prática de atividades físicas em academias. Revista Saúde e Meio Ambiente. 2016; 3(2): 13-22.

26. Ribeiro AF. Taylorismo, fordismo e Toyotismo. Lutas Sociais. 2015; 19(35): 65-79.

\section{Como citar este artigo:}

Morais FM, Lucio Marques Vieira-Souza LMV, Santos JL, Lima Júnior CMA, Costa FB, Silva FJA. Fatores que influenciam a não utilização das Academias ao Ar Livre na cidade de Apucarana-PR. Rev. Aten. Saúde. 2021; 19(68): 363-374. 